\title{
Broadband Excitation and Detection of Cross-Relaxation NMR Spectra
}

\author{
SCOTT D. SWANSON \\ Department of Radiology, University of Michigan, Ann Arbor, Michigan 48109 \\ Received May 14, 1991; revised July 29, 1991
}

Nuclear magnetic resonance cross-relaxation spectroscopy maps the proton solidstate NMR spectrum onto the proton water NMR signal of aqueous heterogeneous materials ( $1-3$ ). Application of a relatively long, low-intensity RF pulse, off resonance from the water Larmor frequency but within the linewidth of the solid, partially saturates the nuclear magnetism of the solid protons. Magnetic cross relaxation between the immobile and mobile protons transfers the saturation of the solid protons to the water protons, decreasing the magnitude of the water NMR signal. A cross-relaxation spectrum is obtained by saturating the immobile protons at many discrete frequencies and plotting the ratio of the water signal with and without RF saturation as a function of the saturation frequency. The intensity and the width of the cross-relaxation spectrum are a function of the longitudinal, transverse, and cross-relaxation rates of the liquid and solid components, the mole fraction of liquid to solid protons, and the magnitude and duration of the saturating RF pulse.

This Communication describes a method for obtaining the entire cross-relaxation spectrum of a spatially homogeneous material with two acquisitions: RF saturation on and RF saturation off. The pulse sequence is demonstrated schematically in Fig. 1. A magnetic field gradient and RF pulse are applied to the sample concurrently. The gradient creates a continuum of frequencies across the sample and establishes at once all conditions of off-resonance saturation for the RF pulse. The decrease in water magnetization by cross relaxation as a function of off-resonance frequency is stored in the sample as a function of the position in the sample and detected by recording a one-dimensional image with the readout gradient applied along the same axis as the frequency-dispersal gradient. The cross-relaxation spectrum is created by dividing the image obtained with RF saturation turned on by one obtained with RF saturation turned off.

The pulse sequence shown in Fig. 1 represents the simplest implementation of broadband cross-relaxation spectroscopy. The sequence may be modified to include slice selection, spin-echo detection, and imaging by phase encoding in either or both of the two spatial dimensions which are not needed to record the cross-relaxation spectrum. In addition the magnitude of the readout gradient may be decreased to lower the bandwidths of the receiver and increase the signal-to-noise of the crossrelaxation spectrum. 


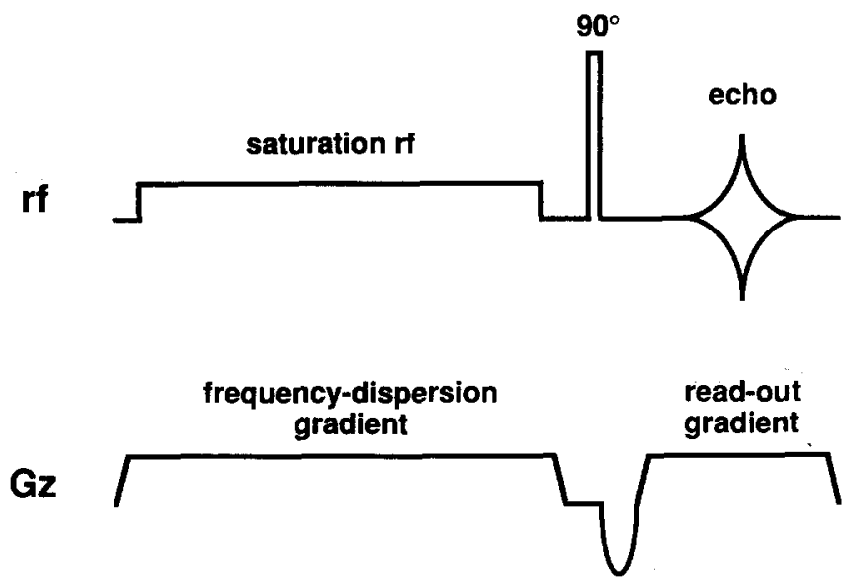

FIG. 1. Pulse sequence for the broadband excitation and detection of cross-relaxation spectra in spatially homogeneous materials.

A comparison of the broadband method with the discrete method was performed to establish that the two methods record the same cross-relaxation spectrum. The results for a sample of cross-linked bovine serum albumin are shown in Fig. 2. Nearly identical spectra are obtained by each method, verifying that both methods do indeed record the same spectrum. Direct RF saturation of water is clearly seen with the broadband method as a narrow Lorentrian lineshape near zero frequency. The width

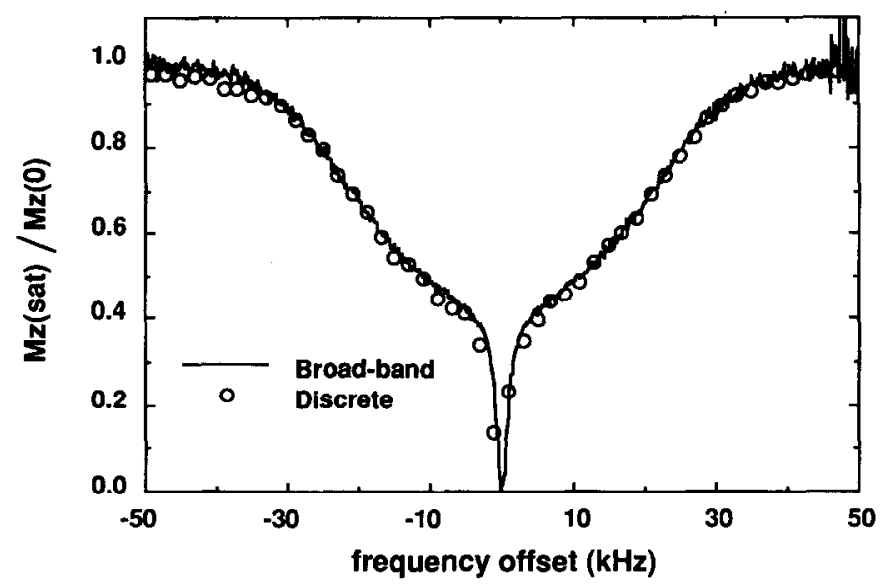

FIG. 2. Comparison of the broadband method of acquiring cross-relaxation spectra with the discrete method on an eight-centimeter-long vial of cross-linked bovine serum albumin. The broadband spectrum was acquired with a saturation RF of $\gamma B_{1} / 2 \pi=700 \mathrm{~Hz}$, a presaturation time of $400 \mathrm{~ms}$, a gradient strength of $3.2 \mathrm{G} / \mathrm{cm}$, a sweep width of $200 \mathrm{kHz}$, a predelay of $2 \mathrm{~s}$, a block size of 1024 (for a spectral resolution of $195 \mathrm{~Hz} /$ point), and an acquisition time of $4.8 \mathrm{~s}$. The point-by-point (discrete) spectrum was acquired using the same parameters as the broadband spectrum but with 128 points at $2 \mathrm{kHz}$ resolution for an acquisition time of $5.12 \mathrm{~min}$. No apodization or signal averaging was done with either signal. 
of this Lorentzian line may be obtained and used to correct for any RF bleedthrough into the cross-relaxation spectrum (4). This work was performed with a GE NMR Instruments $2.0 \mathrm{~T}$ CSI Omega operating at $85.52 \mathrm{MHz}$ for protons and equipped with Acustar S-180 gradients.

Since a single cross-relaxation spectrum can be acquired very quickly, it is not difficult to obtain a series of spectra as a function of the magnitude or duration of the

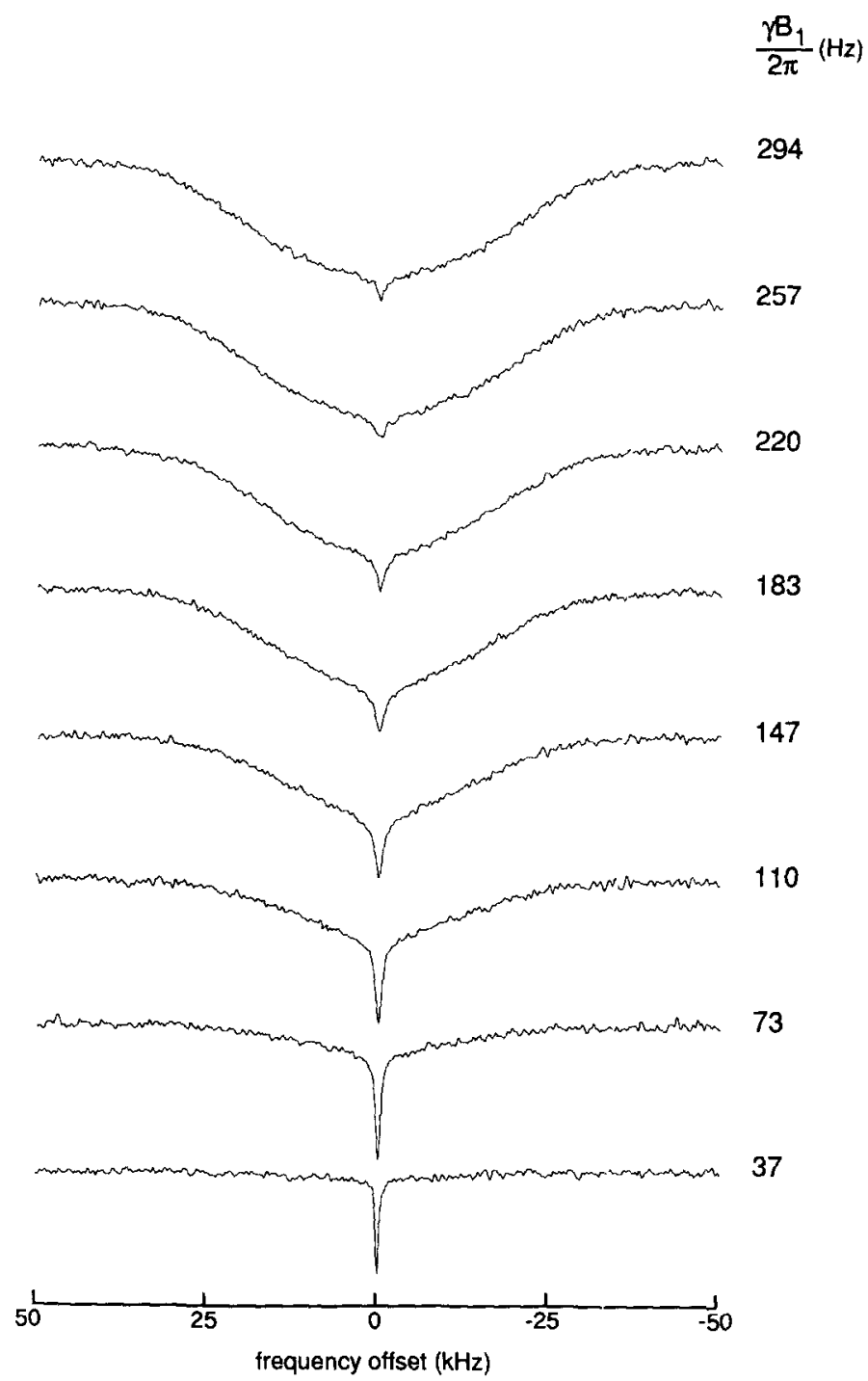

FIG. 3. The cross-relaxation spectrum of hydrated corn starch as a function of the saturation RF power. The spectra were acquired with four acquisitions each, a presaturation time of $400 \mathrm{~ms}$, a gradient strength of $3.33 \mathrm{G} / \mathrm{cm}$, a sweep width of $143 \mathrm{kHz}$, a predelay of $5 \mathrm{~s}$, and a block size of 1024 in a spin-echo experiment with a $\mathrm{TE}$ of $10 \mathrm{~ms}$. 
saturation RF pulse. Figure 3 shows a series of cross-relaxation spectra of hydrated corn starch as a function of the magnitude of the saturation RF pulse. At low values of RF power only direct saturation of the water occurs. As the magnitude of the RF pulse increases, cross relaxation between the liquid and solid components occurs, as seen by the appearance of the cross-relaxation spectrum. It is possible to perform a study of this type and determine an apparent $T_{2}$ of the solid component of the material by measuring the width of the cross-relaxation spectrum as a function of $B_{1}$ field strength (3).

For the broadband technique to work successfully the sample must be spatially homogeneous and large enough for the NMR linewidth of the immobile component to be contained within the bandwidth of the experiment given by $\Delta \nu_{z}=\gamma G_{z} \Delta z$, where $G_{z}$ is the magnitude of the $z$ gradient and $\Delta z$ is the length of the sample. The size of the bovine serum albumin sample in Fig. 2 was $8 \mathrm{~cm}$ and the gradient was $3.2 \mathrm{G} /$ $\mathrm{cm}$, resulting in a bandwidth of $109 \mathrm{kHz}$, which is broad enough to capture the proton solid-state linewidth. With smaller samples, it becomes necessary to increase the gradient strength in order to capture the cross-relaxation spectrum in a single measurement. When the sample size decreases even further, the demand on gradient amplitude will exceed the capability of the hardware. Al that point it will not be possible to obtain the cross-relaxation spectrum in one measurement. However, it is not necessary to revert to the discrete or point-by-point method. Rather, a hybrid of the broadband and point-by-point methods can be constructed, where the frequency of the saturation RF is changed and a number of experiments are performed to cover the proton solidstate linewidth. For example, if the bandwidth of the experiment is $10 \mathrm{kHz}$ as determined by sample size and gradient strength, the broadband sequence outlined in Fig. 1 can be implemented 10 times, with the frequency of the saturation RF changed each time by $+10 \mathrm{kHz}$ to cover the $100 \mathrm{kHz}$ needed to capture the solid-state linewidth. Therefore, the cross-relaxation spectrum can be acquired with high resolution in a piece-by-piece manner. The piece-by-piece acquisition will be of utility in piecewise homogeneous materials.

The cross-relaxation spectrum contains important information about the interactions between, and dynamics of, the solid and liquid components of aqueous heterogeneous material. The technique has applications in biophysics (5), magnetic resonance imaging (6), food science (7), and materials science. The ability to obtain cross-relaxation spectra with high resolution and relative ease will enable detailed studies of magnetic cross relaxation in controlled samples such as hydrated biopolymers and help in the understanding of the observed $T_{1}$ relaxation times measured in heterogeneous materials such as biological tissue.

\section{REFERENCES}

I. J. Grad, D. Mendelson, F. Hyder, AND R. G. Bryant, J. Magn. Reson. 86, 416 (1990).

2. J. GRAD, D. MENDELSON, F. HYDER, AND R. G. BRYANT, Magn. Keson. Med. 17, 452 (1991).

3. J. GRad AND R. G. Bryant, J. Magn. Reson. 90, 1 (1990).

4. J. EnG, T. L. CeCkler, AND R. S. Balaban, Magn. Reson. Med. 17, 304 (1991).

5. H. T. EDZES AND E. T. SAMULSKI, J. Magn. Reson. 31, 207 (1978).

6. S. D. WolfF, J. ENG, AND R. S. Balaban, Radiology 179, 133 (1991).

7. J. Y. WU AND T. M. FADS, Abstracts, 32nd Experimental NMR Conference, St. I ouis, Missouri, p. 140, April 7-11, 1991. 